\title{
Impact of an oral health education intervention among a group of patients with eating disorders (anorexia nervosa and bulimia nervosa)
}

\author{
Laura S. Silverstein ${ }^{1}$, Carol Haggerty ${ }^{2}$, Lattice Sams ${ }^{1}$, Ceib Phillips ${ }^{3}$ and Michael W. Roberts ${ }^{4^{*}}$ (I)
}

\begin{abstract}
Background: It is recognized that eating disorders are serious psychosocial illnesses that affect many adolescents and adults. A pre and post survey study was developed to assess demographics, oral health knowledge and selfimage of patients with eating disorders participating in a hospital-based eating disorder clinic using an original oral health education program. The program's aim is to change the self-image and oral health practices of patients with anorexia-binge eating/purging (AN-BP) and bulimia nervosa (BN) disorders.
\end{abstract}

Methods: A pre-survey was completed by each study participant prior to attending the three educational sessions over a six-week period. A post survey questionnaire was completed after participation in all the educational presentations. Forty-six patients attended all three educational sessions and completed the pre and postquestionnaires.

Results: Most patients knew in advance that AN-BP and BN behavior can cause erosion of the teeth but only 30\% knew the most likely location for the erosion to occur. But, following completion of the educational interventions, $73 \%$ answered the location correctly. Patients who reported going to the dentist regularly were significantly more likely to respond that their teeth/mouth had a positive effect on how they looked to themselves and to others, their general health, and their general happiness. Positive responses to the effect of the teeth/mouth on kissing and romantic relationships were also significantly higher for those who go to the dentist regularly compared to those who do not.

Conclusions: There is a need to further understand AN-BP and BP patients' oral health knowledge and self-image perceptions as it relates to their smile (teeth, mouth) to assist in developing a standardized oral health program for eating disorder centers to implement into their daily curricula. A dental team member in an interdisciplinary eating disorder treatment team is important. Including an oral health education program improves patients' oral hygiene and oral health knowledge, as well as provides a supportive environment to empower the patients to take control of their overall oral health.

Trial registration: This study was retrospectively registered on April 18, 2019 in ClinicalTrials.gov, Identifier: NCT03921632. Keywords: Oral health education, Eating disorders, Anorexia nervosa and bulimia nervosa

\footnotetext{
* Correspondence: mike_roberts16@unc.edu

${ }^{4}$ Division of Pediatric and Public Health, University of North Carolina Adams

School of Dentistry, 228 Brauer Hall CB \#7450, Chapel Hill, NC 27599-7450,

USA

Full list of author information is available at the end of the article
}

(c) The Author(s). 2019 Open Access This article is distributed under the terms of the Creative Commons Attribution 4.0 International License (http://creativecommons.org/licenses/by/4.0/), which permits unrestricted use, distribution, and reproduction in any medium, provided you give appropriate credit to the original author(s) and the source, provide a link to the Creative Commons license, and indicate if changes were made. The Creative Commons Public Domain Dedication waiver (http://creativecommons.org/publicdomain/zero/1.0/) applies to the data made available in this article, unless otherwise stated. 


\section{Plain English summary}

Using a pre and post survey, this study examined the demographics, oral health knowledge and self-image among a group of patients diagnosed with AN-BP or BN. The study participants completed a survey instrument before attending three educational presentations. A post survey questionnaire was completed after the final educational presentation. After participating in the educational presentations, 95\% knew that tooth erosion was the most common oral effect of eating disorders (pre education $=78 \%$ ) and $73 \%$ knew where the erosion most commonly occurred (pre education $=30 \%$ ). Patients who went to a dentist regularly were significantly more likely to report that their teeth/mouth had a positive effect on how they looked to themselves and others, romantic relationships, general health, and their level of happiness. Providing an oral health education program improves the patients' oral health knowledge and empowers them to be proactive in caring for their teeth.

\section{Introduction}

Eating disorders are psycho-social illnesses that affect many adolescents and adults [1]. Individuals with eating disorders can also have additional health issues [2]. Some of these include diabetes, loss of menses in females, heart failure, very low self- esteem, metabolic, cardiovascular and endocrine disturbances [2], and distorted perception of body image [3]. In addition to these systemic problems, oral/dental trauma and dental caries, increase in xerostomia and parotid salivary gland swelling, dental erosion, and periodontal disease have been cited in the literature and are most associated with anorexia-binge eating/purging (AN-BP and bulimia nervosa $(\mathrm{BN})$ [4]. Roberts and $\mathrm{Li}$ stated that the very negative self-perception and self-esteem that many individuals with anorexia nervosa and bulimia nervosa reported could be, in part, a cause for their lack of oral hygiene and increase in dental disease [3].

Often there are general health education programs in eating disorder clinics. However, we are unaware of any established oral health education programs to change and improve patients' oral health behaviors and self-perception as it relates to their smile $[5,6]$. According to the Academy of Eating Disorders, the American Psychological Association, and the American Dental Association, proper oral health care for patients with eating disorders is fragmented. Johnson et al. reported "In addition to patient's lack of knowledge, there is very little evidence, if any, documenting the collaborative interaction between oral health and eating disorder professionals. Individuals who receive treatment for an eating disorder are typically not viewed as needing specialized, preventive oral care and commonly fail to receive appropriate oral health care, or recommendations from the eating disorder treatment team" [7]. There is no indication that these patients have access to the proper oral health education and information. Without adequate oral health education for patients with eating disorders, oral disease can become severe and impact the patient's ability to eat, speak, and socialize [7].

The extent of oral/dental education in curricula within eating disorder treatment centers is unexplored to date $[5,6,8]$. Evidence of patients' self-image as it relates to their smile is also unexplored. The limited studies evaluating perception of oral health knowledge in eating disorder treatment programs suggest the need to further explore provider and client/patient barriers and perception towards oral care [7]. Integrating an oral health program focusing on improving self-image through dental education is essential in providing patients an interdisciplinary approach to care for the entire person. Understanding eating disorder patients' oral health knowledge and perceptions of self-image as it relates to their smile will assist in developing a standardized oral health program for eating disorder treatment centers to implement into their daily curricula.

\section{Methods}

The authors designed a pre and post survey study to assess demographics, oral health knowledge, and selfimage of patients diagnosed with $\mathrm{AN}-\mathrm{BP}$ and $\mathrm{BN}$ participating in a hospital-based eating disorder clinic. The authors consulted and collaborated with eating disorder specialists, dentists, dental hygienists, and psychiatrists in designing the surveys and the education program. An original oral health education program, called Smiles Matter, was created consisting of three different presentations/discussions. Each Smiles Matter session consisted of 15-20 min of didactic learning, $10 \mathrm{~min}$ of a group/personal exercise, and $10 \mathrm{~min}$ of questions that the patients had about their oral health or the topic of the day (Fig. 1).

Patients were consented prior to participating in the study and educational program. A pre-survey was completed by each participant prior to attending the three educational sessions. The presentations were given weekly and addressed general oral health education, esthetics, effects of eating disorders and oral pain, and nutrition for oral health. A post survey questionnaire was completed after the patient had participated in all three educational presentations. A respondent could choose not to answer all questions and some did so. Therefore, the denominator for some questions is different depending upon the number of responses.

The pre-survey consisted of 37 questions and the post survey consisted of 17 questions. Copies of the survey instruments are available from the author. The pre-survey asked questions on the following topics: demographics, oral health knowledge, oral habits and oral health 


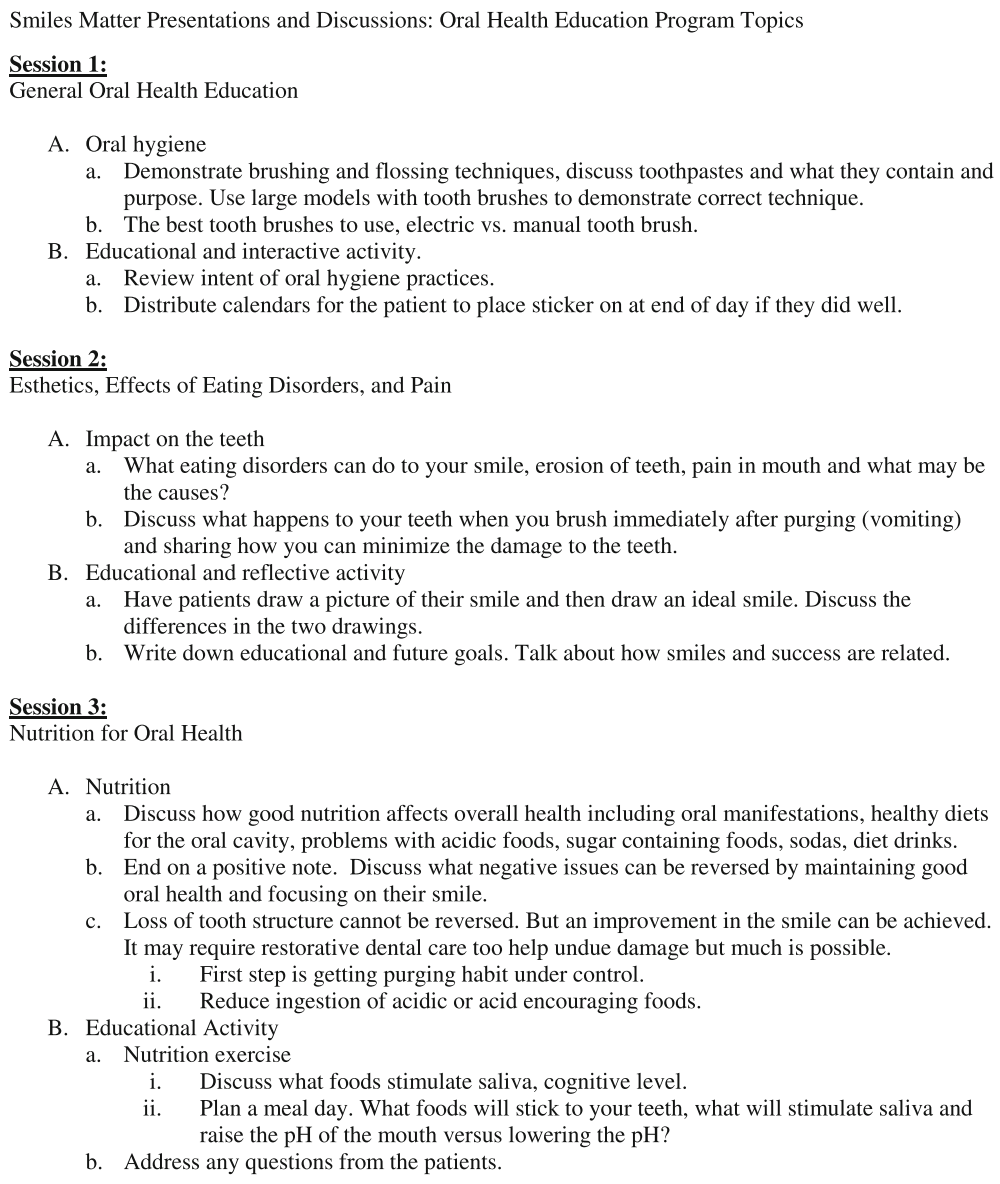

Fig. 1 Smiles Matter Presentations and Discussions: Oral Health Education Program Topics

behaviors prior to diagnosis, oral health and habits since diagnosis with AN-BP or BN and current self-perceptions. The post-survey addressed similar issues after the patients participated in the education program. Chi-square analyses were performed to compare those who completed only the pre-survey questionnaire and those who completed both surveys and to assess the effect of age $(<23$ vs $>=23$ ) and dental visit frequency (regularly vs occasionally/if a problem) on the pre-survey self-perception items. Discordance between pre and post survey responses were analyzed using the McNemar Test or its extension, the Bowken's Test of Symmetry. All analyses were conducted using $\mathrm{SAS}^{\circ} \mathrm{v} 9.4$ software. Level of significance was set at 0.05 for all analyses. Statistical results were not corrected for multiplicity of comparisons because of the nature of the study.

The study design and all questionnaires, presentations, consents to participate and hand-outs were reviewed and approved by the Institutional Review Boards (IRB) at the University of North Carolina at Chapel Hill (IRB \#15-3295). All study sites ceded review responsibility to the UNC-Chapel Hill IRB for management. The study is registered with ClinicalTrials.gov
(Identifier: NCT03921632). Funding for this study was provided by the Department of Pediatric Dentistry.

\section{Study population}

Patients enrolled in three in-patient treatment programs participated in the study; Center of Excellence for Eating Disorders at the University of North Carolina School of Medicine, Carolina House Eating Disorder Treatment Center, and Veritas Collaborative. Patients between the ages of 13 years old and 50 years old were eligible to participate in the study. While there are now eight recognized classifications of eating disorders in the DSM $\mathrm{V}$ [9], this study was limited to patients with anorexia nervosa and bulimia nervosa. Most of the patients who participated in the study did classify themselves as having anorexia nervosa.

\section{Results}

Sixty-seven patients were initially screened (92\% Caucasian $/ 100 \%$ females) and completed the pre-survey questionnaire but only 46 completed both pre- and postquestionnaires and attended all three of the educational module presentations. This was due to the patient being 
released from the clinic or problems with scheduling return visits. There were no statistically significant differences between those who completed the protocol and those who did not (Table 1).

Of those who completed the protocol, the mean age at the time of diagnosis was 21.4 years $(\mathrm{SD}=10.3)$ and at the beginning of the study 25.2 years old $(\mathrm{SD}=10.8)$. The majority (69\%) of the patients had AN-BP. Fiftynine percent reported seeing a dentist regularly but $20 \%$ reported seeing a dentist only when they had a dental problem. Only $15 \%$ of the patients reported being referred to a dentist since their eating disorder was diagnosed. While most patients knew in advance eating disorders behavior can cause erosion of the teeth $(N=35$; $76 \%)$ only $30 \%(N=14)$ knew the most likely location in the mouth for erosion to occur. Eight- $8 \%$ also reported tooth sensitivity as an oral effect of an eating disorder; $78 \%$ reported dry mouth as an effect while $57 \%$ thought salivary gland enlargement and 38\% oral cancer were possible oral effects (Table 2).

Before the intervention, there were no statistically significant differences $(p>0.06)$ in the proportion of positive responses for those less than 23 years of age (54\%) compared to those 23 or older (46\%) with respect to the effect that the teeth / mouth have on self-perception. Patients who reported going to the dentist regularly were significantly more likely to respond that their teeth/mouth had a positive effect on how they looked to themselves $(p=0.03)$, how they looked to others $(p=0.03)$, their general health $(p=0.01)$, and their general happiness $(p<0.001)$ than those who only reported going occasionally, or if they had a problem. Positive responses to the effect of the teeth/mouth on kissing and romantic relationships were also significantly higher for those who go to the dentist regularly compared to those who don't ( $p=0.04$ and 0.002 respectively). Table 2. Sixty-three percent $(N=27)$ of the patients said they had a plan to use the Smiles Matter material. Only $11 \%(N=5)$ did not plan to see a dentist or would go only if they had a problem while $67 \%(N=30)$ planned to see a dentist within 6 months. After participating in the program, $95 \%$ of the patients $(N=41)$ correctly identified dental erosion as the most common dental finding of eating disorders. Similarly, $73 \%(N=32)$ after the program answered correctly where erosion is most likely to occur in the mouth (Table 3).

\section{Discussion}

Evidence-based communication programs are critical for health professionals managing the prevention, treatment, and post treatment of patients of patients with AN-BP and $\mathrm{BN}$ eating disorders. It is important that oral health education programs be included to assist eating disorder patients improve their self-image and shift their focus to the importance of their smile and oral health. The results of this study suggests improved communication and providing appropriate information to AN-BP and $\mathrm{BN}$ eating disorder patients will help change behaviors

Table 1 Comparison of those who participated in both the pre and the post survey $(N=46)$ and those who only participated in the pre-survey $(N=21)$

\begin{tabular}{|c|c|c|c|c|c|c|c|}
\hline \multirow[t]{2}{*}{ Variable } & \multicolumn{3}{|c|}{ Participated in Both Surveys } & \multicolumn{3}{|c|}{ Completed Only Pre-Survey } & \multirow{2}{*}{$\begin{array}{l}P \\
\text { Value }\end{array}$} \\
\hline & Mean & & SD & Mean & & SD & \\
\hline Age at Entry & 25.2 & & 10.8 & 26.9 & & 10.7 & 0.55 \\
\hline \multirow[t]{2}{*}{ Age at Diagnosis } & 21.4 & & 10.3 & 20.6 & & 8.2 & 0.77 \\
\hline & $\mathrm{N}$ & $\%$ & & N & $\%$ & & \\
\hline Eating Disorder & & & & & & & 0.77 \\
\hline Anorexia nervosa & 31 & 69 & & 13 & 62 & & \\
\hline Bulimia Nervosa & 10 & 22 & & 5 & 24 & & \\
\hline Other & 4 & 9 & & 3 & 14 & & \\
\hline Ever Seen a Dentist & & & & & & & 0.79 \\
\hline No & 1 & 2 & & 0 & 0 & & \\
\hline Within last year & 27 & 59 & & 15 & 71 & & \\
\hline Last 2 years & 10 & 22 & & 3 & 14 & & \\
\hline More than 2 years & 8 & 17 & & 3 & 14 & & \\
\hline Frequency of Dental Visit & & & & & & & 0.93 \\
\hline Regularly & 27 & 59 & & 13 & 65 & & \\
\hline Occasionally & 10 & 22 & & 4 & 20 & & \\
\hline Only if problem & 9 & 20 & & 3 & 15 & & \\
\hline
\end{tabular}


Table 2 Effect of dental visit frequency on correct identification of possible oral effects of eating disorders and positive self-perception before the intervention

\begin{tabular}{|c|c|c|c|c|c|}
\hline \multirow{2}{*}{$\begin{array}{l}\text { Possible effects of } \\
\text { eating disorders }\end{array}$} & \multicolumn{2}{|l|}{ Regularly } & \multicolumn{2}{|c|}{ Occasionally/only if problem } & \multirow[t]{2}{*}{$P$ value } \\
\hline & $\mathrm{N}$ & $\%$ & $\mathrm{~N}$ & $\%$ & \\
\hline Tooth erosion & 22 of 26 & 84 & 13 of 20 & 65 & 0.45 \\
\hline Probable erosion sites & 10 of 26 & 38 & 4 of 20 & 20 & 0.33 \\
\hline Tooth sensitivity & 24 of 26 & 92 & 14 of 20 & 70 & 0.37 \\
\hline Dry mouth & 20 of 26 & 81 & 13 of 20 & 66 & 0.99 \\
\hline Enlarged saliva glands & 16 of 26 & 62 & 8 of 20 & 40 & 0.53 \\
\hline Oral cancer & 12 of 26 & 46 & 4 of 20 & 20 & 0.21 \\
\hline No pain & 14 of 26 & 54 & 7 of 20 & 35 & 0.02 \\
\hline \multicolumn{6}{|c|}{ Positive self-perception responses } \\
\hline Confidence & 15 of 26 & 58 & 5 of 20 & 25 & 0.15 \\
\hline Look to others & 15 of 26 & 58 & 3 of 20 & 15 & 0.03 \\
\hline Kissing & 12 of 26 & 46 & 2 of 20 & 10 & 0.04 \\
\hline General health & 17 of 26 & 65 & 5 of 20 & 25 & 0.01 \\
\hline Attendance & 7 of 26 & 27 & 2 of 20 & 10 & 0.54 \\
\hline Success & 7 of 26 & 27 & 4 of 20 & 20 & 0.60 \\
\hline Smiling/laughing & 19 of 26 & 73 & 7 of 20 & 35 & 0.12 \\
\hline Looks to themselves & 15 of 26 & 58 & 4 of 20 & 20 & 0.03 \\
\hline Social life & 10 of 26 & 37 & 3 of 20 & 15 & 0.18 \\
\hline Enjoy eating & 14 of 26 & 54 & 6 of 20 & 30 & 0.27 \\
\hline Speech & 14 of 26 & 54 & 5 of 20 & 25 & 0.10 \\
\hline Choice of foods & 8 of 26 & 30 & 4 of 20 & 20 & 0.17 \\
\hline Enjoy Life & 14 of 26 & 54 & 3 of 20 & 15 & 0.08 \\
\hline Romantic relationship & 10 of 26 & 37 & 1 of 20 & 5 & 0.002 \\
\hline General happiness & 11 of 26 & 42 & 0 of 20 & 0 & $<0.001$ \\
\hline Weight & 4 of 26 & 15 & 2 of 20 & 10 & 0.37 \\
\hline
\end{tabular}

and improve their oral health. The intent of this study was to survey the effectiveness of an original oral health education program, "Smiles Matter", which aimed to improve patient's oral hygiene practices and oral health knowledge, and provide a supportive environment to empower them to take control of their oral health as well as their general health. Throughout the study, participating patients verbally shared that they had not previously had specialized, preventive oral care or recommendations from their eating disorder treatment team targeted to oral health.

It is important that eating disorder patients have access to a dental home to help provide a supportive environment for their oral health, their self-image as it relates to their smile, and their general health. The study found that patients who went to the dentist more frequently had a more positive response to how their teeth affected their lives. It is important for eating disorder treatment centers to provide an oral health educational program and to include an oral health educational program in their treatment protocol.

The primary limitation of this study was the small sample size. It was a challenge to identify patients who were available to complete all components of the study due to the intensity, length, and location of the eating disorder treatment programs. The dropout rate (patients not completing the entire program) was another limiting factor. While additional history was probably recorded in the patient's clinical record (e.g. duration of illness, psychiatric comorbidity) this information was not recorded/analyzed in the present study.

\section{Conclusions}

While statistical significance were shown in this study, the need to further understand eating disorder patients' oral health knowledge and perceptions of self-image as it relates to their smile (teeth, mouth) is important to assist in developing a standardized oral health program 
Table 3 Impact of "Smiles Matter" program

\begin{tabular}{|c|c|c|c|c|c|c|}
\hline Plan to See a Dentist & \multicolumn{2}{|l|}{$\mathrm{N}$} & \multicolumn{4}{|l|}{$\%$} \\
\hline No & \multicolumn{2}{|l|}{2} & \multicolumn{4}{|l|}{4} \\
\hline Within 6 months & \multicolumn{2}{|l|}{30} & \multicolumn{4}{|l|}{67} \\
\hline Within 1 year & \multicolumn{2}{|l|}{10} & \multicolumn{4}{|l|}{22} \\
\hline Only if a problem & \multicolumn{2}{|l|}{3} & \multicolumn{4}{|l|}{7} \\
\hline \multicolumn{7}{|c|}{ Have a Plan to Use Smiles Matter } \\
\hline No & \multicolumn{2}{|l|}{16} & \multicolumn{4}{|l|}{37} \\
\hline Yes & \multicolumn{2}{|l|}{27} & \multicolumn{4}{|l|}{63} \\
\hline \multicolumn{7}{|l|}{ Change in Knowledge } \\
\hline \multicolumn{7}{|l|}{ Dental Erosion } \\
\hline & \multicolumn{6}{|c|}{ Post "Smiles Matter" } \\
\hline & \multicolumn{2}{|c|}{ Correct } & \multicolumn{4}{|c|}{ Incorrect } \\
\hline Pre "Smiles Matter" & N & $\%$ & N & $\%$ & & $P$ value \\
\hline Correct & 34 & 79 & 1 & 2.3 & $(N=35)$ & 0.03 \\
\hline \multirow[t]{2}{*}{ Incorrect } & 7 & 16 & 1 & 2.3 & & \\
\hline & \multicolumn{4}{|c|}{$(N=41)$} & & \\
\hline \multicolumn{7}{|l|}{ Erosion Location } \\
\hline Correct & 12 & 27 & 2 & 4.6 & $(N=14)$ & 0.001 \\
\hline \multirow[t]{2}{*}{ Incorrect } & 20 & 45 & 10 & 22.7 & & \\
\hline & \multicolumn{5}{|c|}{$(N=32$} & \\
\hline
\end{tabular}

for eating disorder treatment centers to implement into their daily curricula/protocol. Including an oral health education program in eating disorder treatment centers appears to improve patients' oral hygiene and oral health knowledge, as well as provides a supportive environment to empower the patients to take control of their overall oral health. Whether the initial improvement in oral habits and awareness will be maintained over time could not be determined by this study.

\section{Abbreviations}

AN_BP: Anorexia-binge eating/purging; BN: Bulimia nervosa; IRB: Institutional Review Board; UNC: University of North Carolina-Chapel Hill

\section{Acknowledgements}

The authors acknowledge the assistance of Abigail Callahan, Helen May and Samantha Glover in conducting this study.

Dr. Silverstein was a dental student at the University of North Carolina

Adams School of Dentistry when this study was conducted.

\section{Authors' contributions}

LSS assisted in developing the study protocol and the educational interventions, recruited the study participants and drafting the manuscript. $\mathrm{CH}$ assisted in developing the study protocol and the educational interventions. LS assisted in developing the study protocol and the educational interventions. CP was the biostatistician who completed the statistical analysis of the collected data. MWR assisted in developing the study protocol, edited the educational interventions and drafting the manuscript. All authors read and approved the final manuscript.

\section{Funding}

This study was funded by the UNC-ASOD, Department of Pediatric Dentistry, Chapel Hill, NC USA.

\section{Availability of data and materials}

The data supporting the results reported in this article is maintained in the UNC Adams School of Dentistry (UNC-ASOD), Department of Pediatric Dentistry, Chapel Hill, North Carolina USA. Please contact author for data requests.

\section{Ethics approval}

This study was approved by the University of North Carolina Institutional Review Boards (IRB\# 15-3295) and the University of North Carolina at Chapel Hill Individual Investigator Agreement (\#15-3295), Office of Human Research Ethics and Institutional Review Boards.

\section{Consent for publication}

Not applicable.

\section{Competing interests}

The authors declare that they have no competing interests.

\section{Author details}

'Department of Pediatric Dentistry, Children's Hospital Colorado, 1575 N Wheeling Street, Aurora, CO 80045, USA. ${ }^{2}$ Division of Comprehensive Oral Health, University of North Carolina Adams School of Dentistry, Chapel Hill, NC 27599-7450, USA. ${ }^{3}$ Division of Craniofacial and Surgical Sciences, University of North Carolina Adams School of Dentistry, Chapel Hill, NC 27599-7450, USA. ${ }^{4}$ Division of Pediatric and Public Health, University of North Carolina Adams School of Dentistry, 228 Brauer Hall CB \#7450, Chapel Hill, NC 27599-7450, USA.

Received: 2 May 2019 Accepted: 15 August 2019

Published online: 05 September 2019

\section{References}

1. Grilo CM, White MA, Masheb RM. DSM-IV psychiatric disorder comorbidity and its correlates in binge eating disorder. Int J Eat Disord. 2009;42(3):228-34.

2. Hicks TM, Lee J, Nguyen T, La Via M, Roberts M. Knowledge and practice of eating disorders among a group of adolescent dental patients. J Clin Pediatr Dent. 2013;38(1):39-43.

3. Roberts MW, Li S-H. Oral findings in anorexia nervosa and bulimia nervosa: a study of 47 cases. J Am Dent Assoc. 1987;115(3):407-10.

4. Romanos $\mathrm{GE}$, Javed F, Romanos EB, Williams RC. Oro-facial manifestations in patients with eating disorders. Appetite. 2012;59(2):499-504.

5. Kisely S, Quek L-H, Pais J, Lalloo R, Johnson NW, Lawrence D. Advanced dental disease in people with severe mental illness: systematic review and meta-analysis. Br J Psychiatry. 2011;199(3):187-93.

6. DeBate RD, Plichta SB, Tedesco LA, Kerschbaum WE. Integration of oral health care and mental health services: dental hygienists' readiness and capacity for secondary prevention of eating disorders. J Behav Health Serv Res. 2006;33(1):113-25.

7. Johnson L, Boyd L, Rainchuso L, Rothman A, Mayer B. Eating disorder professionals' perceptions of oral health knowledge. Int J Dent Hyg. 2017;15(3):164-71

8. Mental Health. A Report of the Surgeon General. Rockville: US Department of Health and Human Services, Substance Abuse and Mental Health Services Administration, Center for Mental Health Services, National Institutes of Health, National Institute of Mental Health; 1999.

9. Feeding and Eating Disorders. American Psychiatric Association. In: Diagnostic and statistical manual of mental disorders. 5th ed. Arlington: American Psychiatric Publishing; 2013.

\section{Publisher's Note}

Springer Nature remains neutral with regard to jurisdictional claims in published maps and institutional affiliations. 